\title{
LO-Phonon Band Filling by Means of Charge Carriers Accelerated in Electric Fields
}

\author{
R. Raguotis and R. Brazis* \\ Semiconductor Physics Institute of the Center for Physical Sciences and Technology \\ A. Goštauto g. 11, LT 01108 Vilnius, Lithuania
}

\begin{abstract}
Electron and phonon distribution in momentum space is modeled using Monte Carlo method. Calculated LO phonon distribution in zinc-blende GaN deviates dramatically from the thermal equilibrium (Planck) function, and well coincides with the phonon number elucidated from the existing anti-Stokes Raman scattering experiments.
\end{abstract}

PACS: 78.45.+h, 78.30.-j, 42.55.-f, 72.10.-d, 72.20.Ht

\section{Introduction}

Non-thermal phonons attract increasing attention in the theory of electron and hole dynamics in crystals subjected to high electric fields. Of special interest is the population inversion of the bands of lattice vibrations, e.g., increasing number of longitudinal optical (LO) phonons relative to the lower-energy (transverse optical - TO, longitudinal acoustic - LA, or transverse acoustic - TA) modes. Inversion is the necessary condition for constructing the phonon-difference laser [1] the device harnessing lattice vibrations for mid- or far-infrared light generation. The term of phonon laser is sometimes misused for designation of ultrasound generator based on stimulated emission and reflection of mechanical waves [2]. We are interested in such situations when a higher-energy phonon mode transforms to the lower-energy one, and the difference of phonon energies gives rise to photon emission.

Seeking to reveal the conditions of creating the phonon-difference laser, we investigate the changes of phonon distributions among the LO and lower bands under influence of charge carriers accelerated in high electric fields. We use Monte Carlo (MC) method for modeling the carrier and lattice dynamics. Simulation includes charge carrier scattering on LO and LA phonons, piezoelectric and impurity ion potentials. Among the examined crystals ( $\mathrm{Si}$ [3], GaN [4], InSb [5], and others [6]), the covalent-bond $n$-type Si crystals are particularly interesting because LO phonons are accumulated there predominantly due to the $g$-type electron umklapp (electron transfer between the equivalent valleys). The process is characteristic of many other indirect-gap semiconductors. In the direct-gap polar semiconductors, LO

* corresponding author; e-mail: brazis@pfi.lt phonon generation by electrons is more intense because electrons accelerated in electric field can loss all their kinetic energy giving birth to the phonons. Among these semiconductors, $n$-type InSb has been found to exhibit the largest population of LO phonon band in comparison with lower bands. However, there were no experimental data in support of MC simulations.

Recent experiments on the Raman scattering in GaN [7] encouraged us to undertake new simulations, and this article presents the comparison of calculated phonon numbers with these elucidated from the intensity of experimental anti-Stokes lines.

\section{Model}

The phonon distribution function $N_{\boldsymbol{q}}$, i.e. the phonon number in the configuration space unit, enters as a control factor in the rate of electron scattering from the initial state $\boldsymbol{k}$ to the final state $\boldsymbol{k}^{\prime}$. For the polar optical scattering, the rate is [8]:

$$
\begin{aligned}
& \lambda_{\mathrm{LO}}(\boldsymbol{k}, \boldsymbol{q})=\frac{e^{2} \omega_{\mathrm{LO}} m^{* 1 / 2}}{4 \pi \hbar \varepsilon_{0}}\left(\frac{1}{\varepsilon_{\infty}}-\frac{1}{\varepsilon_{\mathrm{s}}}\right) \frac{1+2 \alpha \varepsilon^{\prime}}{\sqrt{2 \varepsilon(1+\alpha \varepsilon)}} \\
& \quad \times G(\boldsymbol{k}, \boldsymbol{k} \pm \boldsymbol{q})\left\{\begin{array}{c}
N_{\boldsymbol{q}} \\
N_{\boldsymbol{q}}+1
\end{array}\right\} \ln \left|\frac{k+k^{\prime}}{k-k^{\prime}}\right| .
\end{aligned}
$$

Here $e$ and $m^{*}$ is the electron charge and effective mass, $\varepsilon_{\infty}$ and $\varepsilon_{\mathrm{s}}$ are optical and static dielectric permittivity, $\varepsilon_{0}$ is vacuum dielectric constant, $G(\boldsymbol{k}, \boldsymbol{k} \pm \boldsymbol{q})$ is the integral of overlap of the initial and final Bloch functions, $\varepsilon$ and $\varepsilon^{\prime}$ is the initial and final state energy, and $\alpha$ is the non-parabolicity factor. The phonon absorption rate is proportional to the phonon number $N_{\boldsymbol{q}}$, and $N_{\boldsymbol{q}}+1$ stands for the phonon emission. When electron kinetic energy increases up to the phonon energy $\hbar \omega_{\mathrm{LO}}$, electron stops, and all its momentum is taken by the phonon: 


$$
\hbar q=\hbar k_{\mathrm{LO}} \equiv \sqrt{2 m^{*} \hbar \omega_{\mathrm{LO}}\left(1+\alpha \hbar \omega_{\mathrm{LO}}\right)} .
$$

In electric fields $E$, the cycles of acceleration repeat with the rate $\lambda_{q}^{\mathrm{em}}=e E / \hbar k_{\mathrm{LO}}$ leading to electron streaming in momentum space. Competing scattering mechanisms (by LA phonons, piezoelectric potential, charged and neutral impurity atoms) act so as to disturb the ideal streaming. Monte Carlo simulation is started by using the MaxwellBoltzmann distribution for electrons and the Planck distribution for phonons

$$
N_{\boldsymbol{q}}(0)=1 /\left[\exp \left(\hbar \omega_{\mathrm{LO}} / k_{\mathrm{B}} T\right)-1\right] .
$$

Here $T$ is the thermal equilibrium temperature. If the stationary state is accessible, i.e., $\partial N_{q}(E) / \partial t=0$, then the phonon number in the $q$-space cell of $\Delta q_{x} \Delta q_{y} \Delta q_{z}$ between $\boldsymbol{q}$ and $\boldsymbol{q}+\Delta \boldsymbol{q}$ is

$$
N_{\boldsymbol{q}}(E)=\left(\lambda_{\boldsymbol{q}}^{\mathrm{em}}-\lambda_{\boldsymbol{q}}^{\mathrm{ab}}\right) \tau_{\mathrm{ph}} n\left(\frac{2 \pi}{\Delta \boldsymbol{q}}\right)^{3}+N_{\boldsymbol{q}}(0) .
$$

Here $n$ is electron density, $\tau_{\mathrm{ph}}$ is the phonon lifetime [9], and $\lambda_{\boldsymbol{q}}^{\mathrm{em}, \mathrm{ab}}=\lambda_{\boldsymbol{q}}^{\mathrm{em}, \mathrm{ab}}(\boldsymbol{q}, E, T)$ is the average rate of phonon emission (absorption).

The fraction of momentum space containing approximately $60 \times 60 \times 60$ cells of the size of $0.15 k_{\mathrm{LO}}$ is found to be sufficient for mapping electron and phonon distributions.

Here is the set of GaN parameters [6b] used in MC simulations: $\varepsilon_{\infty}=5.47, \varepsilon_{s}=10.4, \hbar \omega_{L O}=92 \mathrm{meV}$; acoustic deformation potential: $12 \mathrm{eV}$, sound velocity: $4.57 \mathrm{~km} / \mathrm{s}$, piezoelectric constant: $0.5 \mathrm{C} / \mathrm{m}^{2}$, mass density: $6.1 \mathrm{~g} / \mathrm{cm}^{3}$, ionized impurity density $N_{i}=$ $10^{17} \mathrm{~cm}^{-3}$, electron density $n=1.25 \times 10^{18} \mathrm{~cm}^{-3}$ (it is the value deduced from the area density $n_{s}=2.5 \times$ $10^{13} \mathrm{~cm}^{-2}$ of electrons confined to $200 \mathrm{~nm}$ GaN layer at AlN barrier in experiment [7]); electron effective mass $m^{*}=0.193$, nonparabolicity parameter $\alpha=0.21 \mathrm{eV}^{-1}$. The upper valleys $\mathrm{X}$ - and L-valleys have been included as well, although electron inter-valley transfer turned out to be negligible in electric fields accessed in the experiment [7]. LO phonon lifetime is set there to $4.2 \mathrm{ps}$.

\section{Results and discussion}

LO phonon distribution function (Fig. 1) deviates dramatically from the thermal-equilibrium one. Phonon accumulation takes place in the limited momentum-space pocket that is shifted from the Brillouin zone center along the field direction. The main maximum of the phonon distribution function takes place at $q \geq k_{\mathrm{LO}}$. In the vicinity of $q=0$ the phonon number remains at its thermal equilibrium because the low-momentum phonons are scarcely emitted by only high-energy electrons, that are few, and the low-momentum phonon reabsorption rate is high.

Electron distribution does not change significantly under the influence of the phonon accumulation (Fig. 1), therefore the question arises on experiments that could manifest adequately pronounced effect. In physical optics, the phonon number enters linearly in the first-order

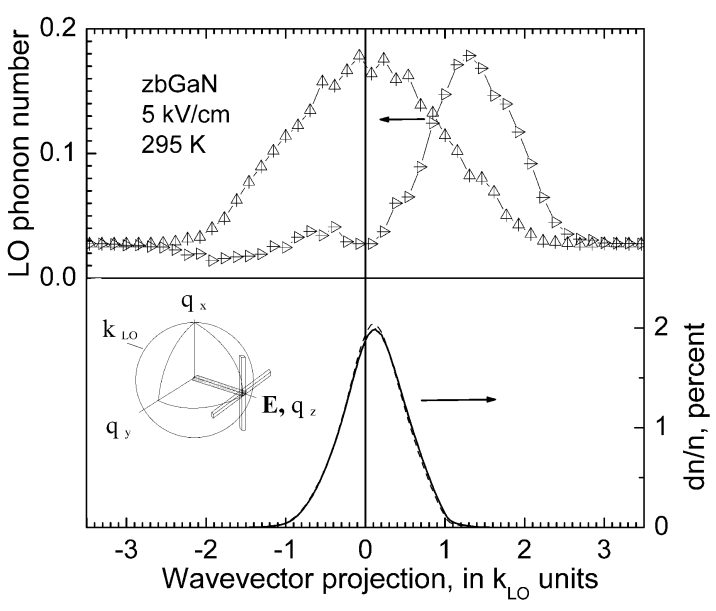

Fig. 1. Monte Carlo data on the LO phonon number distribution in the wavevector space of zincblende GaN crystals (horizontally dashed triangles refer to the tube parallel to the field $\boldsymbol{E}$, and vertically dashed ones stand for the transverse tube, as shown in the inset), as well as electron number $\mathrm{dn} / \mathrm{n}$ distribution over the tube along the field $\boldsymbol{E}$ supposing the excess LO phonon lifetime to be zero (dashed line) and 2 ps (solid line).

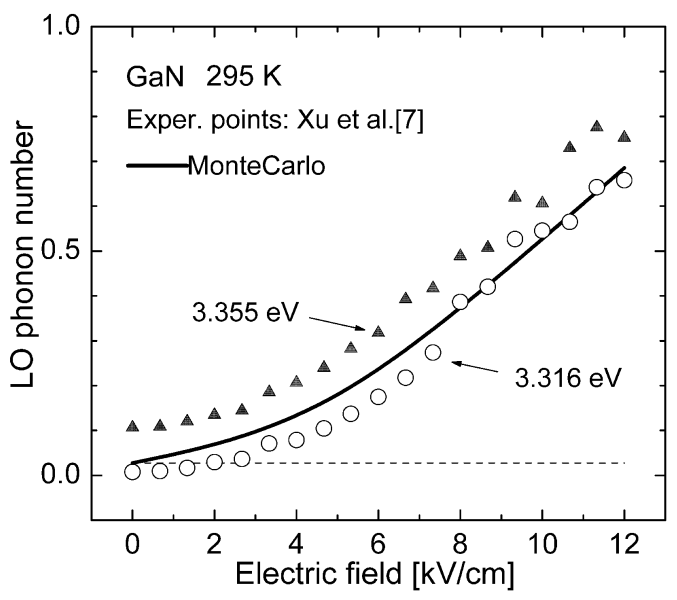

Fig. 2. LO phonon number dependence on electric field at room temperature in zincblende $\mathrm{GaN}$ obtained by Monte Carlo method (solid line) and experimental data (points) reconstructed from the first-order anti-Stokes Raman scattering Ref. [7]; the Raman probing photon energy values are shown at the data points, and the thermal equilibrium number is shown in dashed line.

Raman scattering probabilities: the Stokes line intensity is

$$
I\left(\tilde{\nu}_{0}-\tilde{\nu}_{\mathrm{LO}}\right)=R_{\mathrm{S}}\left(N_{\mathrm{LO}}+1\right),
$$

and anti-Stokes line intensity is

$$
I\left(\tilde{\nu}_{0}+\tilde{\nu}_{\mathrm{LO}}\right)=R_{\mathrm{aS}} N_{\mathrm{LO}} .
$$

Here $\tilde{\nu}_{0}$ is the laser light frequency, $\tilde{\nu}_{\mathrm{LO}}$ is the LO phonon frequency (in GaN crystals it is $\tilde{\nu}_{\mathrm{LO}} \approx 740 \mathrm{~cm}^{-1}$ ), $R_{\mathrm{S}}$ is 
the coefficient that equals numerically to the Stokes line spectral intensity at the LO phonon number $N_{\mathrm{LO}} \rightarrow 0$, and $R_{\mathrm{aS}}$ is the coefficient that equals numerically to the anti-Stokes line spectral intensity for $N_{\mathrm{LO}}=1$. The absolute values of these coefficients depend both on the phonon-photon coupling constants and on the particular experimental apparatus function. Equation (6) shows that the first-order anti-Stokes Raman scattering intensity maximum is for the maximum value of $N_{\mathrm{LO}}$ in the phonon distribution. We refer to experiments by $\mathrm{Xu}$ et al. [7] presenting to our knowledge the first study on the anti-Stokes Raman scattering in GaN. We compare the influence of electric field strength on the maximum value of LO phonon distribution function (Fig. 1) with the dependence deduced from the first-order Raman scattering anti-Stokes experimental line intensity.

$\mathrm{Xu}$ et al. presented actually the "phonon temperature" equivalent for a given value of phonon number. Reformulating their data in terms of phonon number with the use of Eq. (3), we have found that the experimental data obtained at the probing photon energy values of 3.316 and $3.355 \mathrm{eV}$ (Fig. 2) are in good agreement with the results of MC simulation. Experimental phonon number is higher than the calculated one for higher values of the probing photon energy. This can be the effect of excessenergy electrons that are generated by the laser even in the absence of steady electric field.

In future experiments, using increased spectral resolution, one could obtain not only the maximum phonon number but also detailed shape of the phonon distribution in momentum space. Another key question is whether electromagnetic wave emission is possible due to the LO phonon accumulation and their transformation to lower-energy lattice vibration modes. Anticipated electromagnetic wave radiation range would cover mid-infrared (LO-TA transitions in GaN) and far infrared or terahertz ( $\mathrm{LO}-\mathrm{TO}$ in GaN), as discussed in Ref. [10].
Other crystals could candidate for covering gaps not accessible for GaN.

\section{Acknowledgments}

The work is partially supported by the Lithuanian Agency for International Science and Technology Programs in the framework of the COST MP0805 Action.

\section{References}

[1] R. Brazis, Infrared Phys. Technol. 36, 45 (1995).

[2] I.S. Grudinin, H. Lee, O. Painter, K.J. Vahala, Phys. Rev. Lett. 104, 083901 (2010).

[3] R. Brazis, R. Raguotis, Int. J. Infrared Millimeter Waves 22, 845 (2001).

[4] R. Brazis, R. Raguotis, AIP Conf. Proc. 893, 149, (2007); Phys. Status Solidi C 6, 2674 (2009).

[5] R. Brazis, R. Raguotis, Opt. Quant. Electron. 40, 249 (2008).

[6] R. Brazis, D. Nausewicz, R. Raguotis, (a) Phys. Status Solidi B 244, 1662 (2007); (b) J. Phys., Conf. Series 193, 012031 (2009).

[7] G. Xu, S.K. Tripathy, X. Mu, Y.J. Ding, K. Wang, Y. Cao, D. Jena, J.B. Khurgin, Appl. Phys. Lett. 93, 051912 (2008).

[8] R. Mickevičius, A. Reklaitis, Solid State Commun. 64, 1305 (1987); J. Condens. Matter 1, 9401 (1989).

[9] B.K. Ridley, J. Phys., Condens. Matter 8, L511 (1996).

[10] R. Brazis, in: Proc. NATO Advanced Research Workshop on Terahertz and Mid Infrared Radiation: Basic Research and Applications "TERA-MIR 2009", Eds. M.E. Pereira, I.A. Sukhoivanov, IEEE Photonics Soc. and Institute of Theoretical and Applied Physics, Turunç-Marmaris 2009, p. 13. 